\title{
Are YouTube videos a sufficient resource for informing patients in the treatment of rotator cuff tears?
}

\author{
(DLevent Umur ${ }^{1}$, DSerkan Sürüc ̈̈ ${ }^{2}$ \\ ${ }^{1}$ Acıbadem Kadıköy Hospital, Department of Orthopaedics and Traumatology, İstanbul, Turkey \\ ${ }^{2}$ University of Missouri Kansas City, Department of Orthopaedics and Traumatology, United States of America
}

Cite this article as: Umur L, Sürücü S. Are YouTube videos a sufficient resource for informing patients in the treatment of rotator cuff tears? J Health Sci Med 2022; 5(1): 99-103.

\begin{abstract}
Background: The aim of this study was to evaluate the information quality of YouTube videos on rotator cuff tear treatment. Material and Method: A YouTube search was performed using the keyword 'rotator cuff tear' to determine the first 100 most watched videos related to rotator cuff tears. A total of 57 videos met our inclusion criteria and were included in the study. Videos were evaluated for information quality by using DISCERN, the Journal of the American Medical Association (JAMA), and rotator cuff informational assessment (RCIA) scores. Number of views, time since upload, view rate, number of likes, number of dislikes used to calculate the video power index (VPI) and these criteria were used to determine video popularity. Video length (sec), video source and video content were also evaluated and used for correlation evaluations.

Results: The mean DISCERN score was 33.81 (21-56), the mean JAMA score was $3.05(1-4)$, and the mean RCIA score was 3.63 (0.5-7.5). Statistical analysis revealed that, independent of the video source and popularity, the videos were informationally poor and inadequate. The only significant correlation was between video length and data source.

Conclusion: This present study demonstrated that the quality of information provided by YouTube videos about rotator cuff tear treatment was poor. The generation of survey systems for informational videos and the provision of accurate and thorough informational videos by professional health organizations will be the best ways to inform patients.
\end{abstract}

Keywords: Rotator cuff tear, YouTube, video information quality, patient education

\section{INTRODUCTION}

Patients above the age of 60 are more likely to suffer from rotator cuff injuries (1). Its incidence gradually increases with the increasing age of patients (2). In individuals over the age of 80 , the rotator cuff tear incidence is more than $50 \%$ (3). Rotator cuff tears can be asymptomatic, and in some patients, they may also cause symptoms such as severe limitation of motion and pain (4). While conservative treatment is sufficient in asymptomatic patients, surgical treatment is required in symptomatic patients (5).

On the internet, there are several videos designed to inform people about rotator cuff repair. YouTube is one of the platforms where the most videos are uploaded and viewed on the internet (6). Many doctors and healthcare organizations share informational videos and almost all patients seeks for online videos as a second opinion. But this tendency raised a concern about the quality and accuracy of the medical informational videos hosted by YouTube is not a peer-reviewed platform (7). For this reason, the number of likes and views of the videos can create a quality video perception of the patients and cause false information (8). In addition, videos prepared for commercial purposes with commercial concerns may have negative consequences for the treatment of patients (9).

The purpose of this study was to assess the quality of YouTube videos on the diagnosis and treatment of "Rotator Cuff Tears." The evaluation was done from the perspective of a patient seeking medical information.

\section{MATERIAL AND METHOD}

This study was conducted as a YouTube research, there is no need for ethics committee approval.

Videos available on YouTube on 3 November 2020 were scanned using the keyword "Rotator Cuff Tear". The first 100 videos we encountered after the search were evaluated. Non-English, advertising content, less than 1 minute and longer than 20 minutes were excluded from the study. 
The first fifty-seven most watched videos meeting the appropriate criteria were included in our study.

The total number of visits, comments, likes, dislikes and the time interval after upload were recorded. Additionally, video popularity was determined using the video strength index (VPI) values [(likes/dislikes) $\left.{ }^{\star} 100\right]$. We also recorded the video's duration (seconds), provider, and contents.

Two different orthopedic physicians analyzed the videos twice at different times using the DISCERN, Journal of the American Medical Association (JAMA), and Rotator Cuff Informational Assessment (RCIA) scoring systems.

The DISCERN scoring system is composed of 3 sections and has a total of 16 questions. These three sections include 8 questions concerning information's reliability, 7 questions about treatment information, and a question about the overall quality of information (Table 1).

The JAMA scoring system assigns 1 point to each of four criteria (Authorship, Attribution, Disclosure, and Currency), for a total of 4 points. According to the JAMA scoring system, the least valuable information is valued 1 point and the most valuable information is valued 4 points (Table 2).

RCIA is a novel scoring system that consists of 7 sections (definition and pathoanatomy, risk factors and associated conditions, signs and symptoms, imaging, nonoperative treatment, operative treatment and complications), and the overall RCIA score ranges between 0 and 10 (Table 3).

The relationships between VPI and DISCERN, JAMA, and RCIA scores, VPI and video source, video duration and DISCERN, JAMA, and RCIA scores, and view rates and DISCERN, JAMA, and RCIA scores were studied. To avoid misinterpretation related to the age of the videos, the view rate (total number of views divided by time since upload) was used for statistical analysis rather than the total number of views.

Variables were defined using descriptive data (mean, standard deviation, standard error, minimum, median, and maximum for data that showed normal distribution, and median, minimum, maximum, and IQR for data that did not show normal distribution). Pearson's correlation coefficient was calculated to evaluate the relation between normally distributed continuous variables. Due to the nonnormal distribution of the parameters, the Kruskal-Wallis test was used to compare groups and the Mann-Whitney U test (with Bonferroni's correction) to identify the group that caused the difference. Intergroup differences were compared using the one-way ANOVA test. IBM SPSS Statistics 22.0 was used to conduct the statistical analysis. The statistical significance level was set at 0.05 .
Table 1. DISCERN scoring system

\begin{tabular}{ccccccc} 
& No & \multicolumn{3}{c}{ Partially } & & Yes \\
\cline { 2 - 6 } & 1 & 2 & 3 & 4 & 5
\end{tabular}

Section-1: Is the publication reliable?

1 Are the aims clear?

2 Does it achieve its aims?

3 Is it relevant?

Is it clear what sources of

4 information were used to

compile the publication (other

than the author or producer)?

Is it clear when the information

5 used or reported in the

publication was produced?

6 Is it balanced and unbiased?

Does it provide details of

7 additional sources of support and information?

8 Does it refer to areas of uncertainty?

\section{Section-2: How good is the quality of} information on treatment choices?

9 Does it describe how each treatment works?

10 Does it describe the benefits of each treatment?

11 Does it describe the risks of each treatment?

12 Does it describe what would

2 happen if no treatment is used?

Does it describe how the

13 treatment choices affect overall quality of life?

Is it clear that there may

14 be more than one possible treatment choice?

15 Does it provide support for shared decision-making?

\section{Section-3: Overall rating of the publication?}

Based on the answers to all of the above questions, rate the

16 overall quality of the publication as a source of information about treatment choices.

\section{Table 2. JAMA quality assessment}

Authorship Authors and contributors, their affiliations, and Authorship relevant credentials should be provided

References and sources for all content should be

Attribution listed clearly, and all relevant copyright information should be noted

Website "ownership" should be prominently and fully

Disclosure disclosed, as should any sponsorship, advertising, underwriting, commercial funding arrangements or support, or potential conflicts of interest Currency $\begin{aligned} & \text { Dates when content was posted and updated should } \\ & \text { be indicated }\end{aligned}$
. 


\begin{tabular}{|c|c|c|c|c|}
\hline \multicolumn{5}{|c|}{ Rotator Cuff Informational Assessment } \\
\hline No & Criteria & Sub-criteria & Score & Total Score \\
\hline \multirow{3}{*}{1} & \multirow{3}{*}{ Definition \& Pathoanatomy (Max 2 pts) } & Cause (Degenerative/Trauma) (1 pt) & & \\
\hline & & Tear Morphology (Full-Partial) (1 pt) & & \\
\hline & & Rotator Cuff Function $(0.5 \mathrm{pt})$ & & \\
\hline \multirow{4}{*}{2} & \multirow{4}{*}{ Risk Factor \& Associated Conditions (Max 1 pt) } & Age $(0.5 \mathrm{pt})$ & & \\
\hline & & Biceps Tendon Pathology (0.5 pt) & & \\
\hline & & Subacromial Impingement $(0.5 \mathrm{pt})$ & & \\
\hline & & Calcific Tendonitis (0.5 pt) & & \\
\hline \multirow{2}{*}{3} & \multirow{2}{*}{ Signs \& Symptoms (Max 1 pt) } & Pain (Overhead-Night) (0.5 pt) & & \\
\hline & & Loss of Active ROM (0.5 pt) & & \\
\hline \multirow{2}{*}{4} & \multirow{2}{*}{ Imaging (Max $1 \mathrm{pt}$ ) } & MRI (1 pt) & & \\
\hline & & X-Ray (0.5 pt) & & \\
\hline \multirow{3}{*}{5} & \multirow{3}{*}{ Non-Operative Treatment (Max $1 \mathrm{pt})$} & $\operatorname{PTR}(0.5 \mathrm{pt})$ & & \\
\hline & & NSAID $(0.5 \mathrm{pt})$ & & \\
\hline & & Subacromial Injection $(0.5 \mathrm{pt})$ & & \\
\hline \multirow{4}{*}{6} & \multirow{4}{*}{ Operative Treatment (Max 2 pts) } & Arthroscopic Repair (0.5 pt) & & \\
\hline & & Mini-Open Repair (0.5 pt) & & \\
\hline & & Subacromial Decompression $(0.5 \mathrm{pt})$ & & \\
\hline & & Tendon Transfer $(0.5 \mathrm{pt})$ & & \\
\hline \multirow{5}{*}{7} & \multirow{5}{*}{ Complications (Max 2 pts) } & Recurrence/Repair Failure (0.5 pt) & & \\
\hline & & Prognosis $(0.5 \mathrm{pt})$ & & \\
\hline & & Nerve Injury $(0.5 \mathrm{pt})$ & & \\
\hline & & Infection $(0.5 \mathrm{pt})$ & & \\
\hline & & Joint Stiffness $(0.5 \mathrm{pt})$ & & \\
\hline
\end{tabular}

\section{RESULTS}

The results of this study revealed that majority of the evaluated videos (40 videos, 70\%) consists of general information about rotator cuff injuries. 12 videos were about treatment options (21\%), 3 videos were lectures (5\%) and remaining 2 videos classified as other (3\%). Of the 57 videos evaluated, 43 (75.4\%) were uploaded by health channels, 13 (22.8\%) were uploaded by physicians, $1(1.7 \%)$ was uploaded by other sources.

The mean video length was 402.68 seconds (144-1055 seconds), the mean number of views was 78840.98 (29116740), the mean time since the video was posted was 1948.94 days (18-3975 days), the mean daily view rate was 41.96 (0.11-733 per day), the mean number of comments was $30.08(0-428)$, the mean number of likes was 380.89 (0-5600), the mean number of dislikes was 29.05 (05236), and the mean VPI value was 91.92 (57.14-100). The mean scores for RCIA, JAMA and DISCERN were 3.63 (0.5-7.5), 3.05 (1-4) and 33.81 (21-56) respectively.

There was positive, weak, non-significant significant relationship between VPI and video source $(r=0.107$, $\mathrm{p}=0.517$ ), positive, weak, insignificant relationship between VPI and RCIA $(r=0.223, p=0.464)$ for physicians, positive, weak, signicant relationship between video length and JAMA ( $\mathrm{r}=0.417, \mathrm{p}=0.001)$ overall and positive, weak insignificant relationship between VPI and RCIA $(\mathrm{r}=0.049, \mathrm{p}=0.765)$ for health channels.

\section{DISCUSSION}

The Internet is accepted as an unlimited source of information, but since almost none of the sources are peerreviewed, the accuracy of the provided information is a big question. Patients have a growing tendency to investigate their medical conditions and treatment methods. YouTube is one of the most prominent online social media platforms, containing videos on virtually anything, including diseases and their treatment methods (8). Poorquality health information may lead to false expectations, doctor-patient conflicts and cause mistrust. Recently, these topics were evaluated by other researchers. These studies stated that patients have some technical information about their diagnosis and treatment and that they can obtain this information from the internet (10). Although patients' access to this information is an advantage in terms of awareness, it has many disadvantages $(11,12)$. We evaluated the videos in our study by searching YouTube for videos tagged with rotator cuff tears.

DISCERN and JAMA scores are validated scoring systems which were widely used in this kind of studies. On the other hand, these scores were not designed for video sources (DISCERN) and patient information media (JAMA). Therefore we used a novel and unvalidated scoring system, RCIA, specific to rotator cuff injuries. All aspects of rotator cuff injuries are covered including etiology, treatment and complications. Like similars in 
the literature, we think that these kind of scoring systems are essential to evaluate informational media sources.

Considering the data in the literature, it was found that the videos with animation content and shorter duration were more liked and watched (13). In our study, we found that the videos that were shorter and shared by doctors were more liked by the audience. Similarly, Çelik et al. (14) found that video duration was negatively correlated with VPI but positively correlated with quality scores. In addition, we investigated whether there was a correlation between the liking rate and the quality of the videos, and it was determined that there was no significant correlation.

When we conducted a literature review, we discovered several recent studies evaluating the quality of videos used in the diagnosis and treatment of a variety of diseases. Each of these studies determined that the videos lacked a certain level of quality $(15,16)$. In our study, 2 different researchers analyzed videos and scored their video quality using 3 different scoring systems. What the results all had in common was the poor informational quality of the videos. Although most of the videos were prepared by health institutions and a small portion by doctors, the video quality was similarly poor in both groups.

The main reason for the inadequate videos seems to be commercial concerns. Most of the videos were prepared according to the practice of the provider. Since there are no doctor-patient responsibility obligations, most of the providers do not feel obligated to inform the viewers of all aspects of the disease and treatment methods. This leads to a misunderstanding that a specific method could be the only solution. This lack of adequate information reaches its highest point in complications. There was almost no information about the complications patients might face during their treatments. The point that informational videos avoid informing about possible complications of the treatment modality was also mentioned in previous but other than rotator cuff studies $(17,18)$.

Three of the videos included in our study were lectures, and they have the highest mean DISCERN (52) and RCIA (7.5) scores, but they also have the highest mean duration (894 sec.). These three videos have the lowest view rates. Although they are more informative than almost all the other videos, because of the length and amount of highacademic information they contain, viewers do not seem to prefer these videos. This tendency was also reported in a study by Kuru et al. (19) and found a negative correlation between video quality and number of likes which might reflect that high-quality videos are not as popular as low-quality videos.

When we analyzed the statistical results between the study parameters, we found that longer video length correlates only with structural data of the videos like
JAMA quality score. Although interobserver reliability was found to be high for all three scoring systems, there was no correlation between the scoring systems.

Social media platforms are undeniable informational sources today. We think that health professionals and professional health organizations are responsible for developing a suitable way of providing adequate and true health information sources. This could be accomplished by creating a survey system like DISCERN for video sources and providing video-graphic content prepared for patients which includes all aspects of diseases, treatment choices and complications.

This study has some limitations. Only English language videos included in the study and limited to available videos on the exact search date. Number of included videos might be another limitation but since first 100 videos were scanned, this number is beyond the numbers of an average YouTube user search limits. Additionally, an unvalidated tool, RCIA score, used to evaluate content quality but high reliability may refer adequate design.

\section{CONCLUSION}

As a result, content quality of rotator cuff tear videos on YouTube is low. Physicians should inform the patients about insufficient and misleading information sources. Hippocrates stated, "There is no disease but the patient" and treatment methods are chosen based on the patient, not the disease. The correct choice of this treatment procedure depends on the physician-patient relationship with full informed consent. We believe that the patient's misunderstanding may jeopardize this relationship and lead patients to inappropriate treatments ending up with unwanted outcomes.

\section{ETHICAL DECLARATIONS}

Ethics Committee Approval: This study was conducted as a YouTube research, there is no need for ethics committee approval.

Referee Evaluation Process: Externally peer-reviewed.

Conflict of Interest Statement: The authors have no conflicts of interest to declare.

Financial Disclosure: The authors declared that this study has received no financial support.

Author Contributions: All of the authors declare that they have all participated in the design, execution, and analysis of the paper and that they have approved the final version

\section{REFERENCES}

1. Reilly P, Macleod I, Macfarlane R, et al. Dead men and radiologists don't lie: a review of cadaveric and radiological studies of rotator cuff tear prevalence. Ann R Coll Surg Engl 2006; 88: 116-21. 
2. Lehman C, Cuomo F, Kummer FJ, et al. The incidence of full thickness rotator cuff tears in a large cadaveric population. Bull Hosp Jt Dis 1995; 54: 30-1.

3. Tempelhof S, Rupp S, Seil R. Age-related prevalence of rotator cuff tears in asymptomatic shoulders. J Shoulder Elbow Surg 1999; 8: 296-9.

4. Kelly BT, Williams RJ, Cordasco FA, et al. Differential patterns of muscle activation in patients with symptomatic and asymptomatic rotator cuff tears. J Shoulder Elbow Surg 2005; 14: 165-71.

5. Tashjian RZ. Epidemiology, natural history, and indications for treatment of rotator cuff tears. Clinics in Sports Medicine 2012; 31: 589-604

6. YouTube statistics. https://www.youtube.com/yt/press/statistics. html (accessed on 03.11.2020).

7. Madathil KC, Rivera-Rodriguez AJ, Greenstein JS, Gramopadhye AK. Healthcare information on YouTube: A systematic review. Health Informatics J 2015;b21: 173-94.

8. Goyal R, Mercado AE, Ring D, Crijns TJ. Most YouTube videos about carpal tunnel syndrome have the potential to reinforce misconceptions. Clin Orthop Relat Res 2021; 479: 2296-302.

9. Desai T, Shariff A, Dhingra V, et al. Is content really king? An objective analysis of the public's response to medical videos on YouTube. PLoS One 2013; 8: e82469.

10. Akpolat AO, Kurdal DP. Is quality of YouTube content on Bankart lesion and its surgical treatment adequate? J Orthop Surg Res 2020; 15: 1-5.

11.Hungerford DS. Internet access produces misinformed patients: managing the confusion. Orthopedics 2009; 32: 9.

12. Sechrest RC. The internet and the physician-patient relationship. Clin Orthop Relat Res 2010; 468: 2566-71.

13. Morr S, Shanti N, Carrer A, et al. Quality of information concerning cervical disc herniation on the Internet. Spine J 2010; 10: $350-4$.

14. Celik H, Polat O, Ozcan C, Camur S, Kilinc BE, Uzun M. Assessment of the quality and reliability of the information on rotator cuff repair on YouTube. Orthop Traumatol Surg Res 2020; 106: 31-4.

15. Cassidy JT, Fitzgerald E, Cassidy ES, et al. YouTube provides poor information regarding anterior cruciate ligament injury and reconstruction. Knee Surg Sports Traumatol Arthrosc 2018; 26 $840-5$.

16. Fischer J, Geurts J, Valderrabano V, Hügle T. Educational quality of YouTube videos on knee arthrocentesis. J Clin Rheumatol 2013; 19: 373-6.

17. Mert A, Bozgeyik B. Quality and content analysis of carpal tunnel videos on YouTube. Indian J Orthop 2021; 1-6.

18. Ozdemir O, Diren F, Boyali O, et al. Metric evaluation of reliability and transparency of the videos about carpal tunnel syndrome surgery in the online platforms: assessment of YouTube Videos' content. Neurospine 2021; 18: 363-8.

19. Kuru T, Erken HY. Evaluation of the quality and reliability of YouTube videos on rotator cuff tears. Cureus 2020; 12: e6852. 